\title{
Erratum to: Analysis of transient queues with semidefinite optimization
}

\author{
Takayuki Osogami $^{1}$ • Rudy Raymond ${ }^{1}$
}

\section{Erratum to: Queueing Syst (2013) 73:195-234 DOI 10.1007/s11134-012-9309-7}

We correct Corollary 1 in Osogami and Raymond [1]. In [1], Theorem 1 gives an upper bound on the probability, $\pi_{a, b, \infty}$, that the random walk in one dimension crosses a boundary, $b$, before crossing a boundary, $-a$. Theorem 1 is used to derive Corollary 1 , which gives an upper bound on $\operatorname{Pr}(W(a) \geq y)$, the probability that the maximum waiting time in the first busy period of a GI/GI/1 queue is at least $y$, given that the initial work that the first job sees in the system is $a$.

This upper bound in Corollary 1 is derived on the erroneous basis of $\operatorname{Pr}(W(a) \geq$ $y)=\pi_{a, y, \infty}$, which we here correct to $\operatorname{Pr}(W(a) \geq y)=\pi_{a, y-a, \infty}$. For $y>a$, the corrected upper bound on $\operatorname{Pr}(W(a) \geq y)$ is given by replacing $y$ with $y-a$ in the expression of Corollary 1. For $y \leq a$, we have $\operatorname{Pr}(W(a) \geq y)=1$. Here, we also correct the upper bound on the corresponding expected value, $\mathrm{E}[W(a)]=$ $\int_{0}^{\infty} \operatorname{Pr}(W(a) \geq y) d y$, by integrating the corrected upper bound on $\operatorname{Pr}(W(a) \geq y)$ for $y>a$ and integrating $\operatorname{Pr}(W(a) \geq y)=1$ for $y \in[0, a]$. Specifically, the corrected upper bound is given by adding $a$ into the erroneous upper bound in [1]. Equations (2) and (3) involve the same error as Corollary 1 and need to be corrected in the analogous manner.

To summarize, here we correct Corollary 1 in [1] as follows:

The online version of the original article can be found under doi:10.1007/s11134-012-9309-7.

Takayuki Osogami

osogami@jp.ibm.com

Rudy Raymond

raymond@jp.ibm.com

1 IBM, 1623-14 Shimotsuruma, Yamato-shi, Kanagawa 242-8502, Japan 
Corollary 1 Consider the GI/GI/1 queue. Let $C_{A}$ (respectively, $C_{S}$ ) be the coefficient of variation for the interarrival time (respectively, service time), $\lambda$ be the arrival rate, and $\rho$ be the load of the system. Let $W(a)$ be the maximum waiting time in the first busy period, given that the initial work that the first job sees in the system is a $>0$. Let $\xi \equiv\left(C_{A}^{2}+C_{S}^{2} \rho^{2}\right) /(2 \lambda(1-\rho))$ be Kingman's upper bound on the mean waiting time in steady state. Then

$$
\operatorname{Pr}(W(a) \geq y) \leq \min \left\{1,\left(1+(y-a)\left(\xi+a+\frac{1-\rho}{2 \lambda}\right)^{-1}\right)^{-2}\right\}
$$

and

$$
\mathrm{E}[W(a)] \leq \xi+2 a+\frac{1-\rho}{2 \lambda}
$$

Acknowledgement The authors thank the special interest group on queues of the Operations Research Society of Japan for pointing out the error.

\section{References}

1. Osogami, T., Raymond, R.: Analysis of transient queues with semidefinite optimization. Queueing Syst. Theory Appl. 73(2), 195-234 (2013) 